\title{
CT Imaging Evolution of Novel Coronavirus Pneumonia during Hospitalization
}

\author{
Abdulfatah Hassan Maqul*, Allauddin Barak", Yili Zhang ${ }^{\dagger}$, Youmin Guo \\ Department of Medical Imaging, The First Affiliated Hospital of Xi'an Jiaotong University, Xi'an, China \\ Email:cjr.guoyoumin@vip.163.com,macquul200@gmail.com
}

How to cite this paper: Maqul, A.H., Barak, A., Zhang, Y.L. and Guo, Y.M. (2020) CT Imaging Evolution of Novel Coronavirus Pneumonia during Hospitalization. Open Journal of Radiology, 10, 127-137.

https://doi.org/10.4236/ojrad.2020.103013

Received: May 12, 2020

Accepted: August 2, 2020

Published: August 5, 2020

Copyright @ 2020 by author(s) and Scientific Research Publishing Inc. This work is licensed under the Creative Commons Attribution International License (CC BY 4.0).

http://creativecommons.org/licenses/by/4.0/

\section{(c) (i) Open Access}

\begin{abstract}
Object: To evaluate CT Features on serial thin-section computed tomographic (CT) scans in patients with conventional Novel Coronavirus Pneumonia (COVID-19) for the period during which they remained hospitalized. Methods: In this Retrospective study, we collected clinical information including Laboratory investigations and more importantly we focused on collecting imaging data of these 15 selected patients ( 8 men and 7 women, 22 70 years old, average age $(45 \pm 15))$ with COVID-19 disease. The mean time between the initial and repeat thin-section CT scans was $3.3 \pm 2.1$ days, $7.9 \pm$ 2.1 days, $14.2 \pm 1.3$ days after onset of symptoms in these 15 patients. Three experienced Radiologists reviewed the CT images independently and also in collaboration with each other for complicated or unique cases, for the Imaging characteristics like number and site of lesions, distribution of lesions whether it is lobular, lobar, unilateral or bilateral, and comparing the severity of disease in relation to the CT findings. The CT features were compared using the $\chi^{2}$ test and Fisher's exact probability. Results: All of 15 patients had a history of visit to the endemic center i.e. Wuhan city or came in direct contact with an infected individual. Fever (93.3\%) was the most common symptom. Majority of patients had a normal white blood cell count, and normal lymphocyte count although there were patients with leucopenia and lymphocytopenia. CT images showed predominate Ground-Glass opacities in the initial and repeat CT scans with a percentage of $90.6 \%, 53.9 \%, 44.2 \%$ respectively during the three spaced CT examinations; most patients had bilateral lung involvement $(60 \%, 93.3 \%, 93.3 \%)$, the lesions predominantly involved the posterior $(87.5 \%, 71.9 \%, 76.6 \%)$ and peripheral $(90.6 \%, 84.3 \%, 85.7 \%)$ part of the lungs. There were more consolidation and mixed patterns in
\end{abstract}

\footnotetext{
*Abdulfatah Hassan Maqul (first author).

\#Alauddin Barak (co-first author).

${ }^{\dagger}$ Yili Zhang (co-corresponding author).

${ }^{\ddagger}$ Youmin Guo (corresponding author).
} 
repeat CT scan Versus initial CT scan, the difference was statistically significant $(\mathrm{P}$-values were $<0.001)$. The total lung severity score in the three aforementioned timely spaced CT scans were 36 (3.3 \pm 2.1 days) 63 (7.9 \pm 2.1 days), and 57 (14.2 \pm 1.3 days) respectively. The median percentage of pneumonia lesions of the whole lung volume in three times CT scan was $1.69 \%$ (4.59), 3.47\% (5.46), 2.33\% (4.75) respectively. Besides, "Tree-in-bud" sign, lung cavitation, and lymphadenopathy were absent. Conclusion: The Thin-slice Section CT Imaging features show uniquely different characteristics, each time the scans are taken. The most common findings in our study were bilateral multiple peripheral and mostly posterior ground-glass opacities (GGO), however the CT scan images that were taken in a timely manner to follow up demonstrated some remarkable changes, which undoubtedly play an important role in the diagnosis and management of the patient with COVID-19 disease.

\section{Keywords}

Novel Coronavirus Pneumonia (COVID-19), CT Features, Follow-Up Studies

\section{Introduction}

In December 2019, an epidemic of coronavirus disease (COVID 19) erupted in Wuhan City; China [1] [2] which has quickly spread all across the world and was declared as a global health emergency by WHO [3]. The number of patients is rapidly increasing outside of China. The World Health Organization (WHO) named the virus as SARS-COV-2 (severe acute respiratory syndrome coronavirus 2), and the infection it causes was termed as COVID-19 (Coronavirus disease-19) [4] the continuous expansion of COVID-19 has created a pandemic [5]. As of April 14, 2020, 1,844,863 confirmed and 117,021 deaths cases have been reported in Globally, China 83,696 confirmed and 3351 deaths cases have been reported [6].

According to the novel coronavirus pneumonia diagnosis and treatment plan ( $6^{\text {th }}$ trail version) issued by the National Health Committee of the People's Republic of China [7], COVID-19 is divided into four categories Mild, Moderate, Severe and dangerous. Although viral nucleic acid detection using real-time polymerase chain reaction (RT-PCR) remains the standard Laboratory test for the diagnosis of coronavirus disease, the fact cannot be ignored that the timely fashioned CT imaging studies is one of the core reference indexes in the process of COVID-19 diagnosis and treatment plan [8] [9] [10]. Since the majority of COVID-19 cases outside of Wuhan area are usually of Mild to Moderate nature, meaning that symptoms of this type of patients are not serious and the disease is itself not highly infectious and contagious [11], the purpose of this study is to retrospectively analyze the early CT imaging characteristics and then the follow up timely CT scans will clarify short-term evolution of Mild to Moderate COVID-19, to provide auxiliary decision-making for the diagnosis and treatment. 


\section{Data and Methods}

\subsection{General Information}

The study was approved by the medical ethics committee of the First Affiliated Hospital of Xi'an Jiao tong University, and the informed consent was taken from patients or their relatives. The clinical and imaging data of COVID-19 patients in our hospital since the end of January were analyzed retrospectively and a certain group of patients were selected for the study.

Inclusion Criteria:

1) All the patients selected were confirmed as COVID-19.

2) Only patients who had positive symptoms of COVID-19, and had undergone thin layer CT scan images showing typical findings of the disease were included.

3) The selected patients did not have any other viral infection (e.g.: C.A.P.)

4) All selected patients were recently diagnosed and were still under treatment during the course of the study.

Exclusion criteria:

1) Patients with Poor quality CT images, not meeting the criteria to properly study the images and remark on it.

2) All those patients who have severe disease of other body systems, complicated by COVID-19 were excluded.

A total of 15 patients were selected for the study. With 8 males and 7 females, aged $22-70$ years, with a median age of 44 years.

\subsection{CT Examination Method}

CT examination equipment is Philips 16 Slices CT. All patients were laid in a supine position for the scan, the scanning range was from the thoracic entrance to the cost-phrenic angle. The thickness of the layer was $1 \mathrm{~mm}$ and the layer spacing was $5 \mathrm{~mm}$, the tube voltage was $120 \mathrm{kV}$, with automatic tube current, the matrix was $512 \mathrm{~mm} \times 512 \mathrm{~mm}$, and the standard algorithm was $1.0 \mathrm{~mm}$ thin-layer reconstruction. Lung window: window width $\mathrm{WW}=1500 \mathrm{HU}$, window length $\mathrm{WL}=-800 \mathrm{HU}$. mediastinal window: window width $\mathrm{WW}=400 \mathrm{HU}$, window length $\mathrm{WL}=40 \mathrm{HU}$.

\subsection{CT Interpretation}

Three Senior radiologists with 20, 25, and 35 years' working experience respectively read the CT Images. Complicated cases with unusual findings were often discussed with each other.

Image evaluation includes:

1) Evaluation of lesion nature: The lesions were divided into four types according to GROUND-GLASS OPACITIES, (including pure GGO, GGO with enlarged interlobular septum), CONSOLIDATION, MIXED LESIONS (including GGO, consolidation, fibrosis), and FIBROTIC LESIONS, and other associated signs were recorded. The identification of signs was based on the definition of the Fleischner society [12]. 
2) Evaluation of the distribution of the lesions: The lesions can be Unilateral or Bilateral (depending upon which lung is affected Rt or Lt); its location can also be Anterior, Posterior or central (to the mid-axillary line); the location of the lesion is also divided into Upper lung zones, Middle lung zones and Lower lung zones (based on lobar anatomy of lungs).

3) The severity of the lesion: Anatomically the lungs are divided into five lobes ( 3 on Rt. Side and 2 on Lt. side) although statistically the size of a lesion does not directly relate to the severity of the disease. But, in COVID-19 cases, it is found to be useful. The size of each lesion is taken according to the volume ratio of each lung lobe lesion.

0 Points: no lesions

1 point: lesion volume $=\leq 25 \%$.

2 points: lesion volume $=25 \%-\leq 50 \%$.

3 points: lesion volume $=50 \%-<75 \%$.

4 points: lesion volume $=>75 \%$.

The total score is equivalent the total score of the five lung lobes.

4) Quantitative CT evaluation of the percentage of lesions in the total lung volume: The "digital lung TM" software was used for quantitative calculation, automatic segmentation of the volume of lesions and the total lung volume, and then the percentage of lesions in the total lung volume was obtained.

\subsection{Statistical Methods}

SPSS 22.0 statistical software was used for analysis. Frequency and rate were used for counting data. Normally distributed measurement data were expressed by mean $\pm \mathrm{SD}$, and median (interquartile interval) $[\mathrm{M}(\mathrm{q})]$ for skew distribution. $\chi^{2}$ or Fisher's exact probability method was used for comparison with CT for change of pathological properties. $\mathrm{P}<0.05$ was statistically significant.

\section{Result}

\subsection{Patient Demography and Baseline Characteristics}

AGE: Among the 15 patients, 8 were male (53.3\%), 7 were female and the average age was $45 \pm 15$ years old (46.7\%) LOCALITY: 2 cases $(13.3 \%)$ were local people in Wuhan, 5 cases (33.3\%) had a history of living in Wuhan, 8 cases (53.3\%) had a history of contact with people returning home or confirmed to be infected in Wuhan. SIGNS AND SYMPTOMS: 14 cases (93.3\%) had a fever when they were admitted to hospital, including 1 case $(6.7 \%)$ of high fever $\left(>39^{\circ} \mathrm{C}\right), 5$ cases $(33.3 \%)$ of moderate fever $\left(38.1^{\circ} \mathrm{C}-39^{\circ} \mathrm{C}\right), 8$ cases $(53.3 \%)$ of low fever $\left(37.3^{\circ} \mathrm{C}-38.1^{\circ} \mathrm{C}\right) ; 7$ cases $(46.7 \%)$ with cough and expectoration, and 2 cases $(13.3 \%)$ with fatigue, 3 cases (20\%) each with headache and myalgia, 1 case $(6.7 \%)$ with a sore throat, no case with diarrhea and 1 case $(6.7 \%)$ without obvious symptoms. LABORATORY EXAMINATION: It showed that 4 cases (26.7\%) had low leukocyte count and 5 cases $(33.3 \%)$ had low lymphocyte count, as shown in Table 1. 
Table 1. Novel coronavirus pneumonia (COVID-19) patients' demographic and baseline characteristics $(\mathrm{N}=15)$.

\begin{tabular}{|c|c|}
\hline Parameter & Total (percentage, \%) \\
\hline Average Age (Years) & $45 \pm 15$ \\
\hline \multicolumn{2}{|l|}{ Gender } \\
\hline Male & $8(53.3)$ \\
\hline Female & $7(46.7)$ \\
\hline \multicolumn{2}{|l|}{ Epidemic history } \\
\hline Locals of Wuhan & $2(13.3)$ \\
\hline History of residence in Wuhan & $5(33.3)$ \\
\hline Contact with infected patients & $8(53.3)$ \\
\hline \multicolumn{2}{|l|}{ Symptoms and signs } \\
\hline \multicolumn{2}{|l|}{ Fever } \\
\hline$>39.0^{\circ} \mathrm{C}$ & $1(6.7)$ \\
\hline $38.1^{\circ} \mathrm{C}-39^{\circ} \mathrm{C}$ & $5(33.3)$ \\
\hline $37.3^{\circ} \mathrm{C}-38.1^{\circ} \mathrm{C}$ & $8(53.3)$ \\
\hline Cough, expectoration & $7(46.7)$ \\
\hline Fatigue & $2(13.3)$ \\
\hline Headache & $3(20)$ \\
\hline Myalgia & $3(20)$ \\
\hline Sore throat & $1(6.7)$ \\
\hline Diarrhea & 0 \\
\hline No obvious symptoms & $1(6.7)$ \\
\hline \multicolumn{2}{|l|}{ laboratory examination } \\
\hline Leukopenia & $4(26.7)$ \\
\hline Lymphopenia & $5(33.3)$ \\
\hline
\end{tabular}

\subsection{Changes in Imaging Characteristics}

All 15 patients underwent CT examinations three times. The average time from the first CT examination to the occurrence of symptoms was $3.3 \pm 2.1$ days. The average time from the reexamination to the occurrence of symptoms was $7.9 \pm$ 2.1 days. The average time from the third CT examination to the occurrence of symptoms was $14.2 \pm 1.3$ days.

\subsubsection{Changes in the Number, Distribution, and Severity of Lesions}

In the first CT examination, there were 64 lesions, $6(40 \%)$ patients had unilateral lesions (among which 5(33.3\%) were solitary lesions) and 9 (60\%) patients had lesions distributed along both lung fields. The second CT showed that the number of lesions increased to 89 , only one patient $(6.7 \%)$ still had a single lesion involving one side, and the other 14 patients (93.3\%) showed bilateral lung involvement with multiple distributions. Four patients changed from a single lesion to multiple lesions (Figure 1 \& Figure 2). By the third CT examination, the number of lesions was reduced to 77 , and the main pattern is multiple lesions 

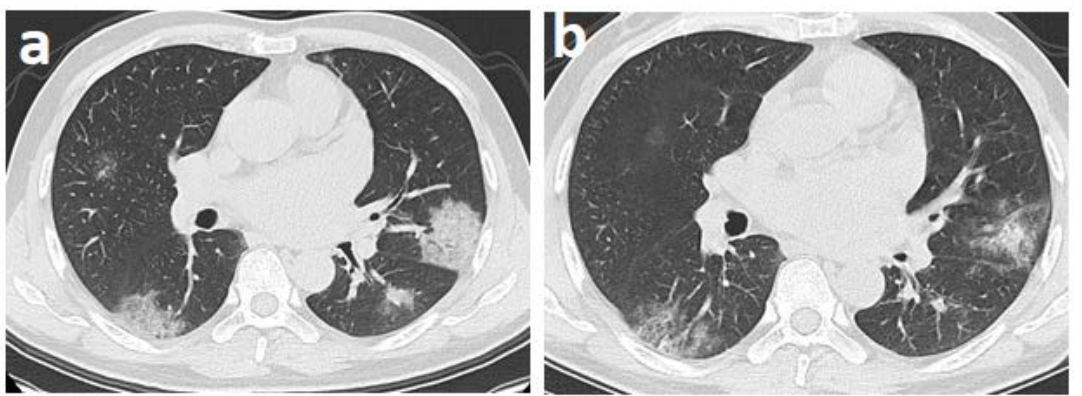

Figure 1. Male, 42 years old, COVID-19. (a) The CT scan showed that there were multiple GGO in both lungs on February 4, 2020. (b) After 6 days, the lesions in both lungs were obviously absorbed and improved and there were multiple linear high-density shadows in the lesion area.
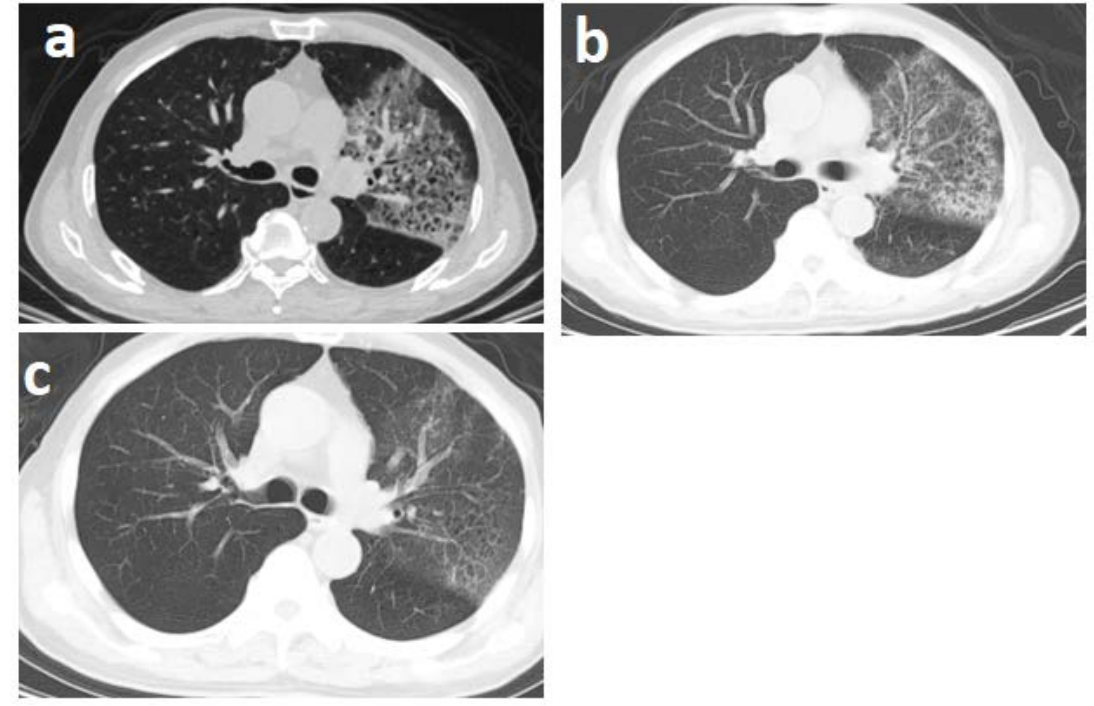

Figure 2. Male, 32 years old, COVID-19. (a) The CT scan showed that in the upper lobe of the left lung, there was a paving stone sign and there were also enlarged blood vessels and dilated bronchus in the lesion on February 9, 2020. (b) There was no change in the size of the lesion, but the density of the lesion decreased on February 15, 2020. (c) There was no change in the size of the lesion, but the lesion was absorbed and the density of the lesion decreased progressively on February 15, 2020.

distributed around both lung fields. In all three CT examinations taken, the lesions were mainly located around the periphery of both lung fields $(90.6 \%$, $84.3 \%, 85.7 \%)$ and the posterior to the mid-axillary line $(87.5 \%, 71.9 \%, 76.6 \%)$ respectively. There is no significant difference in the distribution of the upper and lower lung fields, as shown in Table 2.

Quantitative CT showed that the median proportion of pneumonia lesions to total lung volume was 1.69 (4.59\%) in the first CT examination, 3.47 (5.46\%) in the second CT examination and $2.33(4.75 \%)$ in the third CT examination; the overall score of lung disease severity was 36,63 and 57, respectively, suggesting that the imaging manifestation was aggravating the severity of the lesion around the first week of the course of the disease; the severity of the lesion gradually decreased after the second week, as shown in Table 2. 
Table 2. Novel coronavirus pneumonia (COVID-19) patients were followed up for CT lesions distribution and severity changes $(\mathrm{N}=15)$.

\begin{tabular}{|c|c|c|c|}
\hline CT signs & First $\mathrm{CT}^{\mathrm{a}}$ & Second $\mathrm{CT}^{\mathrm{b}}$ & Third CT \\
\hline Number of lesions & 64 & 89 & 77 \\
\hline \multicolumn{4}{|l|}{ Focus distribution } \\
\hline One lung involvement, $\mathrm{n}(\%)$ & $6(40)$ & $1(6.7)$ & $1(6.7)$ \\
\hline Bilateral lung involvement, n (\%) & $9(60)$ & $14(93.3)$ & $14(93.3)$ \\
\hline Single focus, $\mathrm{n}(\%)$ & $5(33.3)$ & $1(6.7)$ & $1(6.7)$ \\
\hline Two or more multiple lesions, No. (\%) & $10(66.7)$ & $14(93.3)$ & $14(93.3)$ \\
\hline \multicolumn{4}{|l|}{ The dominant distribution area of focus } \\
\hline Anterior, n (\%) & $8(12.5)$ & $25(28.1)$ & $18(23.3)$ \\
\hline Posterior, n (\%) & $56(87.5)$ & $64(71.9)$ & $59(76.6)$ \\
\hline Peripheral, n (\%) & $58(90.6)$ & $75(84.3)$ & $66(85.7)$ \\
\hline Central, n (\%) & $6(9.4)$ & $14(15.7)$ & $11(14.3)$ \\
\hline \multicolumn{4}{|l|}{ Affected lung field } \\
\hline $\begin{array}{c}\text { Upper lung (two upper lobes + right middle } \\
\text { lobe), } \mathrm{n}(\%)\end{array}$ & $27(42.2)$ & $43(48.3)$ & $36(46.8)$ \\
\hline Lower lung, n (\%) & $37(57.8)$ & $46(51.7)$ & $41(53.2)$ \\
\hline $\begin{array}{l}\text { Pneumonia focus as a percentage of total } \\
\text { lung volume }(\%,[M(Q)])\end{array}$ & $1.69(4.59)$ & $3.47(5.46)$ & $2.23(4.75)$ \\
\hline The total score of lung disease severity & 36 & 63 & 57 \\
\hline
\end{tabular}

${ }^{\mathrm{a}}$ The average distance from the first CT examination to the first symptom was $3.1 \pm 2.1$ days; ${ }^{\mathrm{b}}$ The average distance from the second CT examination to the first symptom was $7.7 \pm 2.3$ days; The average distance from the third CT examination to the first symptom was $14.2 \pm 1.3$ days.

\subsubsection{Change of Lesion Nature}

In the early stage of the disease ( $3.1 \pm 2.1$ days after the onset of symptoms), the nature of the lesion on CT is mainly GGO (90.6\%), Consolidation (1.6\%) and mixed lesions $(7.8 \%)$ were few and no fibrotic lesions. By the first week of the disease course ( $7.7 \pm 2.3$ days), the nature of the lesions on CT was still mainly GGO (53.9\%), but Consolidation (29.2\%) and mixed lesions (15.7\%) increased significantly, and 1 patient developed fibrotic Focus. By the second week of the disease (14.2 \pm 1.3 days), GGO lesions continued to decrease (44.2\%), lesions with consolidation (22.1\%) decreased slightly, mixed lesions (22.1\%) and fibrotic lesions (11.7\%) increased (Figure 3). Comparing the changes of lesions in three $\mathrm{CT}$ examinations, except for mixed lesions, the remaining three differences were statistically significant (P-values were $<0.001,<0.001,0.001$ ). The evolution of lesions of different natures is shown in Table 3.

\subsubsection{Other Accompanying Signs}

Pleural effusion in 2 cases (13.3\%), bronchiectasis in 2 cases (13.3\%), no tree-in-bud sign reflecting small airway lesions, no cavity lesions, no obvious 


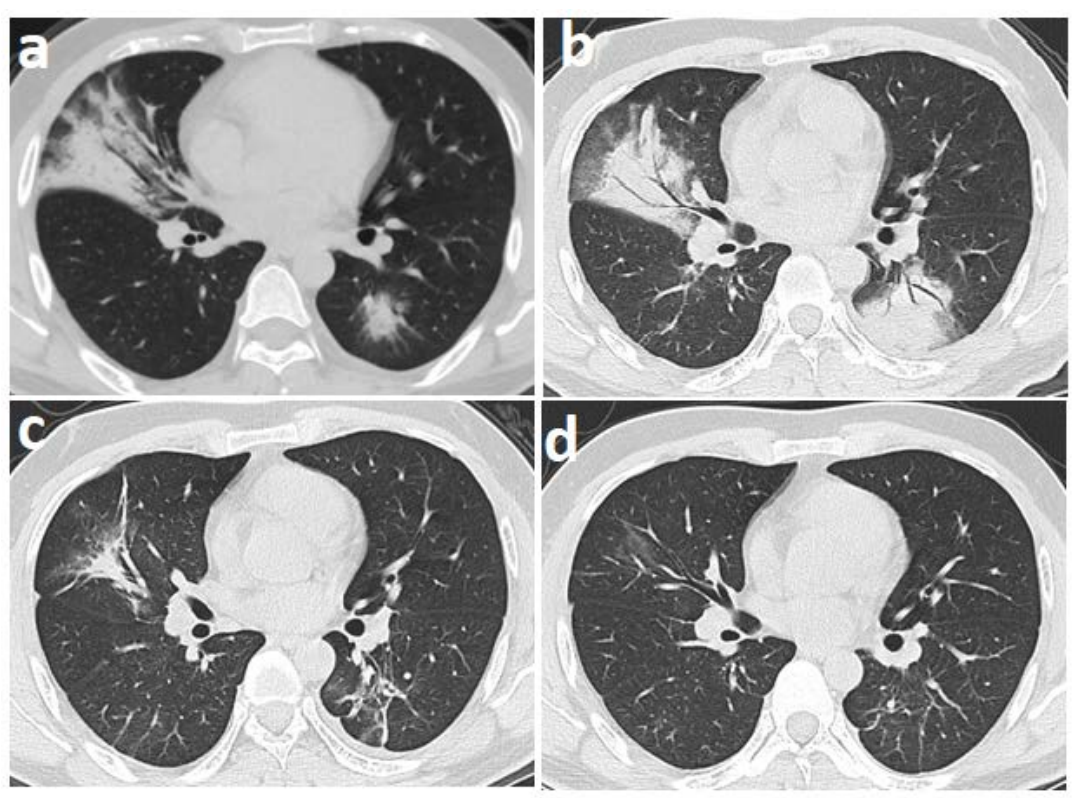

Figure 3. Female, 45 years old, COVID-19. (a) The CT scan showed that there was consolidation in the middle lobe of right lung and lower lobe of left lung on January 29, 2020. (b) The extent of the lesion was enlarged in both lungs on February 1, 2020. (c) The lesions were obviously absorbed and improved and there were multiple linear high-density shadows in the lesion areas on February 12, 2020. (d) All the lesions have been absorbed nearly completely on February 16, 2020.

Table 3. Novel coronavirus pneumonia (COVID-19) patients follow up CT lesion pattern change $(\mathrm{N}=15)$.

\begin{tabular}{cccccc}
\hline Pathological pattern & FirstCT $^{\mathrm{a}}$ & Second CT $^{\mathrm{b}}$ & Third CT $^{\mathrm{c}}$ & Statistical value & $\boldsymbol{p}_{\text {-value }}^{*}$ \\
\hline Total number of lesions & 64 & 89 & 77 & $49.419^{\mathrm{n}}$ & $<0.001$ \\
Ground glass lesions, n (\%) & $58(90.6)$ & $48(53.9)$ & $34(44.2)$ & $34.619^{\mathrm{m}}$ & $<0.001$ \\
Solid lesions, n (\%) & $1(1.6)$ & $26(29.2)$ & $17(22.1)$ & $19.049^{\mathrm{m}}$ & $<0.001$ \\
Mixed lesions, n (\%) & $5(7.8)$ & $14(15.7)$ & $17(22.1)$ & $5.388^{\mathrm{m}}$ & 0.070 \\
Fibrosis focus, n (\%) & 0 & $1(1.1)$ & $9(11.7)$ & $12.696^{\mathrm{n}}$ & 0.001 \\
\hline
\end{tabular}

${ }^{a}$ The average distance from the first CT examination to the first symptom was $3.1 \pm 2.1$ days; ${ }^{b}$ The average distance from the second CT examination to the first symptom was $7.7 \pm 2.3$ days; ${ }^{\circ}$ The average distance from the third CT examination to the first symptom was $14.2 \pm 1.3$ days; ${ }^{\mathrm{m}}: \chi^{2}$ test was used; ${ }^{\mathrm{n}}$ : Fisher exact probability method.

mediastinal and hilar lymphadenopathy were seen.

\section{Discussion}

Similar to the previous reports [13] [14], this study found that at the time of admission, the common type of COVID-19 patients CT imaging mainly showed GGO with multiple, peripheral and posterior lung fields distribution. This sign may mainly reflect the damage at the alveolar level in the early stage of the disease. The pathological study confirmed that [15], the local pathological manifestations of COVID-19 lesions were obvious alveolar damage, including alveolar 
edema, protein, and cellulose exudation, a variety of inflammatory cells and polymorphonuclear cells aggregation in the air containing cavity, and different degrees of alveolar cell proliferation, thickening of the alveolar wall and interlobular septum, thus forming early GGO changes on CT. It has been reported in the literature that the early lesion of patients with COVID-19 is mainly located in the lower lobes of both lungs. This phenomenon is not found in our study, which may be affected by the small sample size of our study, or the previous study samples are mainly local patients in Wuhan.

Compared with the results of three CT examinations in this group of patients, the number of lesions on CT and the severity of lung lesions in COVID-19 patients reached the maximum about one week after symptom onset, and then gradually decreased in the second week. Pan et al. [16] compared the clinical and imaging data of 21 patients with COVID-19 longitudinally, which indicated that the most serious lesion was found in the patients about 10 days after the onset of the disease. In addition, although the three CT examinations showed GGO as the main lesion $(90.6 \%, 53.9 \%, 44.2 \%)$, the second and third CT examinations showed that GGO lesions decreased gradually, and consolidation and mixed lesions increased significantly, suggesting that consolidation and mixed lesions may be the potential biological markers reflecting the long and short course of disease and image progression of patients with COVID-19 [16] [17]. Despite the progression of the disease, the corresponding clinical symptoms and vital signs of the patients tend to be stable, suggesting that CT signs may have a certain lag compared with the clinical signs of COVID-19 patients. The corresponding relationship between CT signs and clinical symptoms and laboratory examination needs further study.

In previous studies on SARS, it was found that the fibrous cord is the residual sign in the lung after healing [18]. In this study, it was found that the fibrous cord appeared in the short-term in patients with COVID-19, and there were 2 patients with bronchiectasis. The long-term outcome of pulmonary fibrosis in patients with COVID-19 and the correlation with the efficacy need to be further clarified in the follow-up longitudinal study. Although the CT imaging features of COVID-19 have certain characteristics, the early and dynamic changes do not have specificity, so it is difficult to distinguish COVID-19 from influenza A and other viral pneumonia [18] [19]. However, the common signs of community-acquired pneumonia, such as tree-in-bud sign, lymphadenopathy, and cavitation, are absent in CT images, which is helpful to reduce the spectrum of differential diagnosis. If the $\mathrm{CT}$ image of the patient shows multiple ground glass shadows in both lungs, the posterior and prepheral of the lung field distribution, and there is a corresponding epidemiological history, with fever and dry cough symptoms, the white blood cell count and lymphocyte count in the laboratory examination are reduced, COVID-19 should be highly suspected, and nucleic acid (RT-PCR) examination is required for the diagnosis.

Limitations of this study: the sample size is small, and the influence of COPD, 
cardiovascular disease, and other basic diseases on the change of pulmonary lesions is not considered. In addition, the patients included in this study were younger and had an age-related bias, and there was no complete agreement between the first time and the reexamination $\mathrm{CT}$ at the time, which may have some impact on the experimental results.

\section{Conclusion}

The most common findings in our study were bilateral multiple peripheral and mostly posterior ground-glass opacities (GGO), The CT imaging changes show a trend within two weeks after symptoms appear. Compared with clinical symptoms, there is a delay, consolidation and mixed lesions may be potential biological signs of disease development. CT Imaging examination and evaluation are advantageous for the diagnosis and management of COVID-19.

\section{Conflicts of Interest}

The authors declare no conflicts of interest regarding the publication of this paper.

\section{References}

[1] Zhu, N., Wang, W., et al. (2020) A Novel Coronavirus from Patients with Pneumonia in China, 2019. The New England Journal of Medicine, 382, 727-733. https://doi.org/10.1056/NEJMoa2001017

[2] Lu, H., Tang, Y., et al. (2020) The Wuhan SARS-CoV-2-What's Next for China. Journal of Medical Virology, 92, 546-547. https://doi.org/10.1002/jmv.25738

[3] WHO (2020) Statement on the Second Meeting of the International Health Regulations (2005) Emergency Committee Regarding the Outbreak of Novel Coronavirus (2019-nCoV). WHO, Geneva.

[4] WHO (2020) Novel Coronavirus (2019-nCoV) Situation Report-22.

[5] WHO (2020) WHO Director-General's Opening Remarks at the Media Briefing on COVID-19-11 March 2020.

[6] WHO (2020) Infection Prevention and Control during Health Care When COVID-19 Is Suspected.

https://www.who.int/publications-detail/infection-prevention-and-control-duringhealth-care-when-novel-coronavirus-(ncov)-infection-is-suspected-20200125

[7] Diagnosis and Treatment Protocol for Novel Coronavirus Pneumonia (6th Trial Version). http://www.nhc.gov.cn/yzygj/s7653p/202002/8334a8326dd94d329df351d7da8aefc2/ files/b218cfeb1bc54639af227f922bf6b817.pdf

[8] Fang, Y.C., Xie, J.C., Lin, M.J., Ying, L.J., Pang, P.P., Ji, W.B., et al. (2020) Sensitivity of Chest CT for COVID-19: Comparison to RT-PCR. Radiology. https://doi.org/10.1148/radiol.2020200432

[9] Li, X., Liu, B., Yu, Y., et al. (2020) COVID-19 Infection Presenting with CT Halo Sign. Radiology Cardiothoracic Imaging, 2, e200026. https://doi.org/10.1148/ryct.2020200026

[10] Phelan, A.L., Gostin, L.O., et al. (2020) The Novel Coronavirus in Wuhan, China: Challenges for Global Health Governance. JAMA, 323, 709-710. 
https://doi.org/10.1001/jama.2020.1097

[11] Huang, C.W., Li, X., et al. (2020) Clinical Features of Patients Infected with 2019 Novel Coronavirus in Wuhan, China. The Lancet.

[12] Heber MacMahon, D.P.N., Goo, J.M., Lee, K.S., Leung, A.N.C., Mayo, J.R., Mehta, A.C., Ohno, Y., Powell, C.A., Prokop, M., Rubin, G.D., Schaefer-Prokop, C.M., Travis, W.D., Van Schil, P.E. and Bankier, A.A. (2017) Guidelines for Management of Incidental Pulmonary Nodules Detected on CT Images: From the Fleischner Society. Radiology, 284, 228-243. https://doi.org/10.1148/radiol.2017161659

[13] Chung, M., Mei, X., et al. (2020) CT Imaging Features of 2019 Novel Coronavirus (2019-nCoV). Radiology, 295, Article ID: 200230. https://doi.org/10.1148/radiol.2020200230

[14] Shi, H., Jiang, N., et al. (2020) Radiological Findings from 81 Patients with COVID-19 Pneumonia in Wuhan, China: A Descriptive Study. The Lancet Infectious Diseases, 20, 425-434. https://doi.org/10.1016/S1473-3099(20)30086-4

[15] Tian, S., Niu, L., et al. (2020) Pulmonary Pathology of Early Phase SARS-COV-2 Pneumonia, Preprints 2020.

[16] Pan, F., Sun, P., et al. (2020) Time Course of Lung Changes on Chest CT during Recovery from 2019 Novel Coronavirus (COVID-19) Pneumonia.

[17] Kanne, J.P. (2020) Chest CT Findings in 2019 Novel Coronavirus (2019-nCoV) Infections from Wuhan, China: Key Points for the Radiologist. Radiology, 295, 16-17.

[18] Ooi, G.C., Müller, N.L., et al. (2004) Severe Acute Respiratory Syndrome: Temporal Lung Changes at Thin-Section CT in 30 Patients. Radiology, 230, 836-844. https://doi.org/10.1148/radiol.2303030853

[19] Chinese Society of Radiology (2020) Radiological Diagnosis in Novel Coronavirus Pneumonia: Expert Recommendation from the Chinese Society of Radiology (Version 1). Chinese Journal of Radiology, 54, E001. 